\title{
El Valor de la Tecnología: Enfoques Novedosos para su Determinación
}

\author{
The Value of Technology: Novel Approaches for its Estimation
}

\author{
Claudia-Nelcy Jiménez', Oscar Castellanos²
}

\begin{abstract}
Traditionally, Technology valuation has been applied with the financial focus of Project management, focusing on the economical dimension. However, several authors have detected problems in this approach and propose the inclusion of qualitative aspects of technology. The aim of this paper is to identify alternative proposals for estimating the value of technology. A scientometric study was carried out and as a result, novel works were identified, like the use of roadmapping, econometrics and neural networks in technology valuation. We conclude that different approaches exist, but, in general, aspects as the knowledge embodied in technology are not taken into account, with a reductionist and linear view of the valuation process and the technology itself. We formulate some considerations on an approach of technology valuation from biology, as an alternative approximation from the organicistic view, in order to add attributes like dynamicity and adaptation.
\end{abstract}

Keywords: technology valuation; technological capabilities; alternatives of technology valuation; hard and soft technology.

Tradicionalmente la valoración tecnológica se ha abordado con un enfoque financiero de gestión de proyectos, centrándose en la dimensión económica. Sin embargo, diversos autores han detectado falencias en esta aproximación, proponiendo incluir aspectos cualitativos de la tecnología. El objetivo de este artículo es identificar propuestas alternativas a las tradicionales para determinar el valor de la tecnología. Se hizo un análisis cienciométrico, y como resultado se identificaron trabajos novedosos como la aplicación de roadmapping, econometría y redes neuronales en procesos de valoración. Se concluye que, si bien se han planteado aproximaciones diferentes, en general se desconocen aspectos como el conocimiento embebido en la tecnología, con una visión reduccionista y lineal del proceso de valoración y de la propia tecnología. Se plantean consideraciones sobre un abordaje de la valoración desde la biología, como enfoque alternativo a partir de la visión organicista, agregando al proceso atributos como la dinamicidad y la adaptación.

Keywords: valoración tecnológica; capacidades tecnológicas; alternativas de valoración tecnológica, tecnología dura y blanda.

'Profesora de la Facultad de Minas, Universidad Nacional de Colombia.Adress: Calle 44 No. 45-67, Unidad Camilo Torres Bloque B5 Oficina 303, Bogotá, Colombia. Phone: 57-I 3165000 Ext. 10730. E-mail: cnjimenezh@unal.edu.co

2Profesor de la Facultad de Ingeniería, Universidad Nacional de Colombia. E-mail: ofcastellanosd@unal.edu.co

ISSN: 07 I8-2724. (http://www.jotmi.org)

Journal of Technology Management \& Innovation (c) Universidad Alberto Hurtado, Facultad de Economía y Negocios. 


\section{Introducción}

Las organizaciones se apoyan en fuentes de tecnología tanto internas como externas para generar innovaciones, lo que a su vez lleva a transacciones y acuerdos en torno a la tecnología, que hacen cada vez más necesaria la consolidación de procesos para estimar su valor. Así como el factor tecnológico se ha ido convirtiendo en un determinante de la competitividad y su relevancia estratégica es evidenciada por una cantidad mayor de organizaciones, el papel de los procesos para valorarla adecuadamente también se vuelve clave. Como lo señala Hetman (1973), debido al fuerte impacto de la tecnología en la organización y su entorno, es necesario analizar los sistemas sociotécnicos, medir el impacto social de la tecnología, evaluar tecnologías alternativas y estudiar los futuros tecnológicos. No obstante, esto también representa un nuevo desafío en el marco de la gestión tecnológica, ya que debe responder a la complejidad implícita en los paquetes tecnológicos, dada su naturaleza y composición.

La valoración tecnológica es un mecanismo clave en los procesos de toma de decisiones, abarcando tanto los componentes financieros y técnicos, como la gestión estratégica de la tecnología (Li y Chen, 2006). Angelo et al. (2008) afirman que es un objetivo prospectivo conocer el valor de tecnologías específicas para incentivar la transferencia tecnológica. Así mismo, la valoración de una colección de activos relacionados, incluyendo la tecnología, es un pre- rrequisito para determinar si la estrategia de negocios seleccionada está generando una tasa de retorno aceptable (Watkins, 1998).

El objetivo de este artículo es analizar el tema de la valoración tecnológica para identificar enfoques diferentes a los tradicionalmente empleados (como los métodos de costos, ingresos y de mercado) que se orienten a la valoración tomando en cuenta las diferentes dimensiones que la tecnología implica, más allá de su dimensión monetaria y su componente tangible. Entre estos desarrollos se presenta un caso orientado a la valoración de la tecnología tomada en su concepto más amplio que incluye las capacidades tecnológicas organizacionales. Finalmente se plantea una propuesta como vía alternativa de abordaje a partir de elementos y conceptos de la biología, en búsqueda de enriquecer el tópico de la valoración tecnológica, frente a las falencias identificadas en la revisión de literatura que sirve de base para la presente investigación.

\section{Concepto y categorías de valoración tecnológica}

Así como el concepto de tecnología ha cambiado y se ha enriquecido, el de valoración también ha evolucionado como se muestra en la Figura I. Desde la visión Taylorista, la tecnología se consideraba como un medio de producción fundamentalmente tangible, si bien más adelante se empezó a reconocer el papel del conocimiento embebido en estos

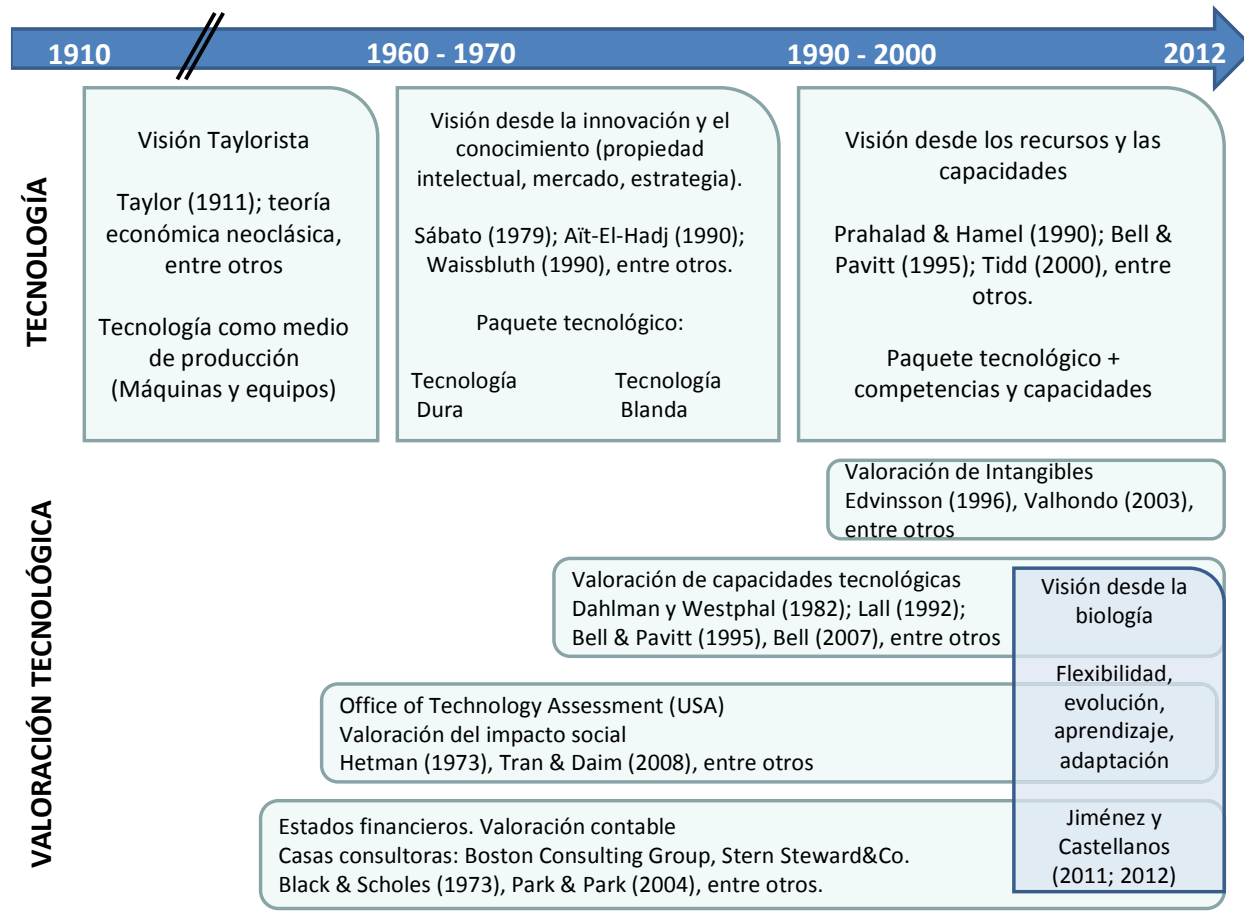

Figura I. Evolución del concepto de tecnología y su valoración. Fuente: elaboración propia

ISSN: 07 I8-2724. (http://www.jotmi.org)

Journal of Technology Management \& Innovation (c) Universidad Alberto Hurtado, Facultad de Economía y Negocios. 
bienes, hablándose de paquetes tecnológicos, hasta llegar una visión ampliada que también incluye las capacidades tecnológicas organizacionales. En este artículo se tomará como referente esta conceptualización amplia de la tecnología.

De forma similar a la definición de tecnología, la valoración tecnológica se ha complementado desde el enfoque puramente contable, al incluir la valoración de los aspectos intangibles, en donde el conocimiento y las capacidades organizacionales han generado aproximaciones diferentes a la monetaria, e involucrar aspectos sociales en la medición del impacto de la tecnología en contextos determinados.

La valoración tecnológica ha sido definida por diversos autores, empero, su concepto académico se comenzó a explorar en años recientes ( $\mathrm{Li}$ y Chen, 2006). Desde una perspectiva fundamentalmente monetaria, estos autores afirman que la valoración de tecnología busca determinar el máximo beneficio económico mediante el uso de información de manera eficaz y con una serie de métodos razonables, es decir, permitir a un comprador o un vendedor beneficiarse mediante la plena utilización de toda la información disponible.

Con una aproximación más amplia, Elói y Santiago (2008) indican que el propósito de la valoración no es predecir el valor exacto de la tecnología, sino proporcionar un valor esperado que capture los riesgos e incertidumbres inherentes al proceso de innovación tecnológica. Estos autores afirman que la valoración y la evaluación tecnológica son procesos complementarios que hacen parte de uno más amplio conocido como comercialización de nuevas tecnologías. Adicionalmente, la valoración se orienta a la determinación del valor intrínseco de un activo tecnológico, a diferencia del pricing o fijación de su precio (LES, 2008).
En general, se evidencia que en la literatura existen diversos conceptos sobre valoración tecnológica, algunos más restringidos que otros, supeditados a la conceptualización de tecnología que se maneje y al contexto en que se desarrolle el proceso de valoración.

\section{Categorías de la valoración tecnológica}

Pueden identificarse tres categorías de acuerdo con la orientación de las técnicas que se reportan en la literatura para valorar la tecnología (Figura 2): una primera categoría agrupa los métodos tradicionales de valoración, enfocados en la tecnología dura y la determinación de su valor económico. La segunda categoría está orientada a los activos intangibles. La última categoría hace referencia a la medición del impacto social de la tecnología.

En la categoría tradicional se encuentran los métodos de costos, de mercado, de ingresos, de opciones reales, entre otros. El primero se basa en el principio económico de sustitución: un inversionista prudente no pagaría por un activo tecnológico más de lo que costaría crear o adquirir un activo similar. Este método es usado generalmente cuando la tecnología se encuentra en las primeras etapas de desarrollo y su aplicación en el mercado no es clara todavía, es decir, cuando el nivel de certeza es muy bajo y el conocimiento del negocio futuro es limitado, por lo cual solo provee un valor aproximado o una referencia aproximada. Por otra parte, el método de mercado estima el valor de un activo a partir del análisis de las ventas u ofertas recientes de activos comparables de otras compañías. El método de ingresos mide el valor de un activo a través del valor presente de sus beneficios económicos futuros (Pavri, 1999; Mard, 2000 citados en Park y Park, 2004). Una mejora al método de ingresos

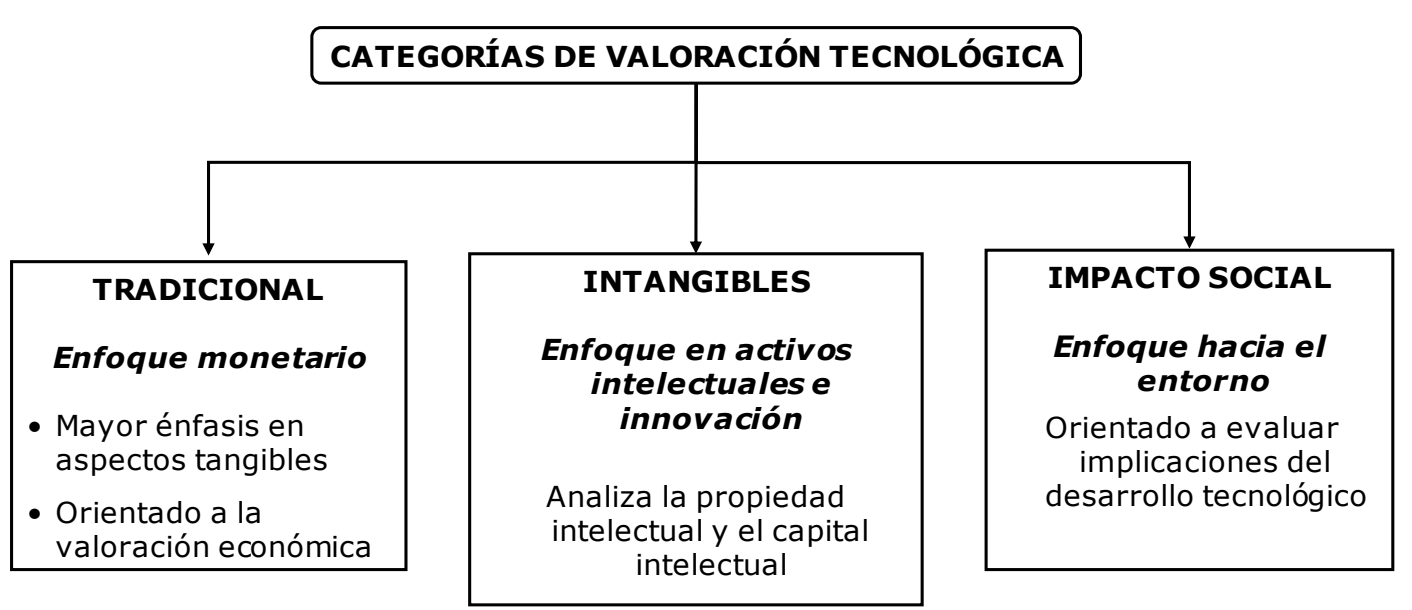

Figura 2. Categorías de la valoración tecnológica. Fuente: elaboración propia

ISSN: 07I 8-2724. (http://www.jotmi.org)

Journal of Technology Management \& Innovation (c) Universidad Alberto Hurtado, Facultad de Economía y Negocios. 
son las opciones reales, enfoque inicialmente propuesto por Black y Scholes (1973), puesto que lo supera al considerar la incertidumbre y el riesgo. Este método ha sido ampliamente estudiado en el marco de la valoración de proyectos, no necesariamente tecnológicos, y se ha considerado el más completo por su flexibilidad. Sin embargo, según Lewis, Enke y Spurlock (2004), la dificultad radica en que la literatura no lo ha abordado para permitir su aplicación directa, ya que se ha enfocado en los cálculos matemáticos y ecuaciones diferenciales, haciéndolo demasiado complejo.

En la categoría de intangibles, se ha reconocido que cualquiera sea la forma de la transacción, su valoración representa un problema común y relevante (Chiesa, Gilardoni y Manzini, 2005), precisamente por esta condición de intangibilidad. Dentro de esta categoría se destaca la valoración del capital intelectual, que incluye modelos como el Navegador de Skandia, una de las primeras compañías en realizar esfuerzos coherentes por medir adecuadamente sus activos de conocimiento, que combina elementos conceptuales y metodológicos con cinco áreas de enfoque: la financiera, del cliente, de proceso, de renovación, y de desarrollo y capital humano (Bontis, 200I;Valhondo, 2003).

La categoría de valoración del impacto social (o Technology Assessment) tuvo sus orígenes en la década de los años sesenta en Estados Unidos, cuando se incrementaron notablemente los efectos de diferentes tecnologías implementadas a gran escala en el común de las personas, con el objetivo principal de mejorar su calidad de vida pero con inevitables efectos colaterales muchas veces indeseados (Hetman, 1973). Sin embargo, como lo señalan Tran y Daim (2008), en la evolución del concepto de valoración del im-

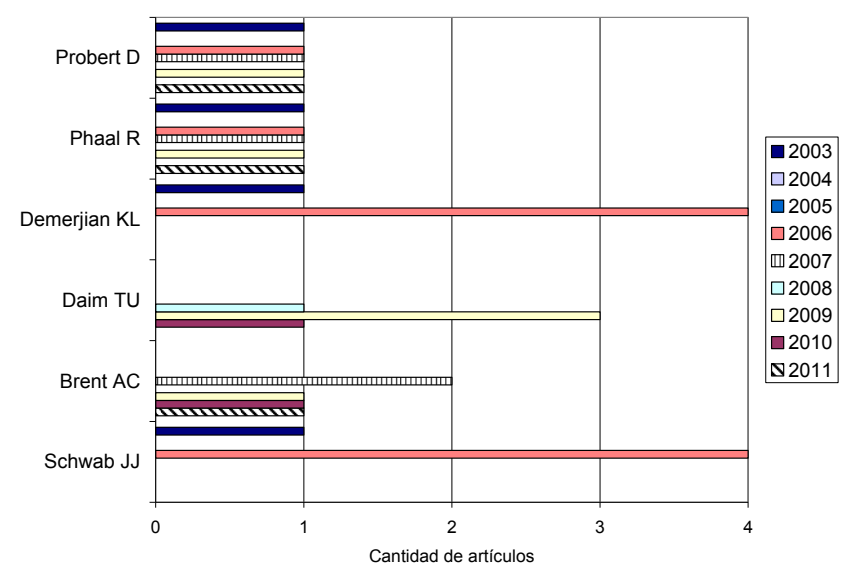

Figura 3.Autores más destacados en el tema de valoración tecnológica. Fuente: información de las bases de datos de artículos ScienceDirect, ISI Web of Knowledge y Scopus, procesada con Excel. Fecha de búsqueda: II de mayo de 20 II. pacto social de la tecnología en el marco de la gestión tecnológica, se ha evidenciado que no solo atañe al campo de las políticas públicas, sino que en el ámbito empresarial se ha asociado con la preparación o disponibilidad tecnológica (technological readiness - ¿cuándo estará lista alguna tecnología particular para alcanzar determinado objetivo o uso en el negocio o en la producción?). Coates y Fabian (citado en Tran y Daim, 2008) encontraron que muchas empresas ven la valoración del impacto social como una forma de anticipación de los efectos del mundo exterior en sus propias actividades, más que anticipar los efectos de sus actividades en el entorno, por lo cual la llamaron "Inverted Technology Assessment". Por su parte, Maloney (1982) afirma que esta categoría de valoración tecnológica en el sector privado es un fenómeno creciente que ha buscado determinar las implicaciones sociales, políticas y económicas relacionadas con el desarrollo tecnológico de la organización, pero en forma diferente a como lo ha hecho el sector público en cuatro dimensiones: objetivos, estructura, tiempos y otras percepciones, $y$ con el propósito de maximizar las ganancias en el corto y mediano plazo.

\section{Enfoques alternativos en la valoración de tecnología}

Al hacer una revisión de la literatura sobre valoración tecnológica se encontró que son varios los autores que han publicado más de cuatro artículos en el tema en la última década (esta revisión se hizo a través de la aplicación de técnicas cienciométricas a las referencias encontradas en las bases de datos ScienceDirect, ISI Web of Knowledge y Scopus, disponibles en el Sistema Nacional de Bibliotecas de la Universidad Nacional de Colombia, con ecuaciones de búsqueda que contenían las palabras clave technology valuation, technology assessment, tecnology appraisal y sus combinaciones con una ventana de búsqueda correspondiente al periodo enero de $200 \mathrm{I}$ - mayo de $20 \mathrm{I}$ I. La búsqueda se realizó el II de mayo de 201 I y se trabajó en total con 317 referencias). De acuerdo con las temáticas abordadas por estos autores, puede afirmarse que dos de ellos (Phaal y Probert) trabajan en temas relativos a la valoración económica tradicional, mientras que los cuatro restantes (Schwab, Dermejian, Brent y Daim) ubican sus desarrollos en la categoría de valoración del impacto social de la tecnología.

Respecto a los autores Phaal y Probert, abordan la valoración económica de la tecnología y su relación e impacto en la gestión tecnológica, pero hacen una crítica a los métodos tradicionales y proponen vías alternativas. Al discutir y evidenciar las desventajas de los métodos de valoración como el flujo de caja descontado frente al riesgo, la incertidumbre y la ambigüedad, plantean un modelo híbrido que consiste en un árbol de decisiones para las primeras etapas de un proyecto de selección de tecnología, en las cuales es frecuente el riesgo específico, y una red binomial para las

ISSN: 07 I8-2724. (http://www.jotmi.org)

Journal of Technology Management \& Innovation (c) Universidad Alberto Hurtado, Facultad de Economía y Negocios. 
etapas posteriores, donde el riesgo de mercado es el factor más importante (Hunt et al., 2003; Shehabuddeen, Probert y Phaal, 2006; Hunt et al., 2007; Thorn et al., 201 I). Así mismo, para enfrentar tales deficiencias en los métodos tradicionales, plantean una visión de la valoración con base en el ciclo de vida de la tecnología y el concepto de madurez de la misma, a partir de entrevistas en empresas del Reino Unido de los sectores de telecomunicaciones, aeroespacial y farmacéutico (Farrukh et al., 2009).

Los autores Schwab y Dermejian investigan en conjunto sobre temas relativos a la valoración del impacto de de emisiones de material particulado en el ambiente de Nueva York en la época de los atentados a las torres gemelas (Ren et al., 2003; Qureshi et al., 2006; Weimer et al., 2006; Dutkiewicz et al., 2006a; Dutkiewicz et al., 2006b; Cowan y Daim, 2009). Por su parte, Brent trabaja en la valoración del impacto de las tecnologías energéticas en regiones africanas, enfatizando en la sostenibilidad mediante el uso de energías renovables (Nyoka y Brent, 2007; Brent y Kruger, 2009; Brent y Rogers, 20 I 0; Musango y Brent, 20 I la; Musango y Brent, 20 I l b).

Adicionalmente, Daim (Tran y Daim, 2008; Daim e Intarode, 2009; Daim et al., 2009; Daim y Cowan, 2010) ha trabajado en la definición del marco conceptual, los métodos y herramientas de la valoración del impacto social de la tecnología (Technology Assessment), así como en la aplicación del roadmapping para la valoración de energías renovables y tecnologías limpias. Este puede identificarse como un enfoque alternativo que aún está siendo explorado por algunos autores. En el caso de Daim, su trabajo se orienta al estudio de las tecnologías para la generación de electricidad, con énfasis en el papel de las energías renovables en la reducción de costos y daños ambientales, por lo que el roadmapping se emplea para predecir las tendencias en dicha reducción y en el potencial de crecimiento de la generación de energía, lo cual es aplicable a la planeación nacional y regional de este tipo de recursos (Cowan y Daim, 2009).

Entre otros trabajos que han empleado el roadmapping en la valoración tecnológica, principalmente en su categoría de valoración del impacto social, se encuentra el de Fleischer Decker y Fiedeler (2005), que se enfoca en prever y evaluar los impactos a largo plazo de la nanotecnología. Así mismo, Robinson y Propp (2008) examinan el estudio de la ciencia y la tecnología emergentes a través de metodologías de análisis tecnológico orientado al futuro (FTA por sus siglas en inglés) entre las cuales está el roadmapping, aplicado en la Red Europea de Investigación en Nanotecnología. Por otra parte, Koivisto et al. (2009) proponen la integración de metodologías FTA, como el roadmaping, con herramientas de evaluación de riesgos en proyectos relacionados con infraestructura, creación de negocios y cambio climático.
Tomando en cuenta las palabras clave de los artículos sobre valoración tecnológica revisados para este artículo, se identificaron otros trabajos que pueden considerarse como enfoques alternativos a los comúnmente empleados en esta temática. Hilty et al. (2006) proponen una metodología basada en dinámica de sistemas combinada con la planeación por escenarios para simular los efectos negativos de las tecnologías de información y comunicación (TIC) en la Unión Europea en el año 2020. Janssen y Mendys-Kamphorst (2007) estudian un modelo económico para representar los cambios en los mercados dominados por tecnologías antiguas cuando se introduce una nueva tecnología y existen externalidades de red, suponiendo que la calidad de la tecnología puede ser medida objetivamente y que los consumidores difieren en su valoración de dicha calidad. Tavana y Sodenkamp (2010) emplean lógica difusa para la evaluación y selección de tecnologías informáticas y computacionales en el Centro Espacial Kennedy, que incluye el empleo de un modelo de análisis de decisiones multicriterio conocido como Fuzzy Euclid, así como un método de defusificación para obtener resultados cuantificables. El trabajo de Dubin (2007) desarrolla un método econométrico para la valoración de una marca, con el cual se estima la demanda de los bienes con dicha marca y se compara con la de productos similares sin marca y commodities. Finalmente, se encontró un trabajo en el que se aplican redes neuronales para construir un modelo de valoración monetaria tanto de patentes como de las compensaciones por daños que deben exigirse en caso de que los derechos asociados sean transgredidos (Lai y Che, 2009).

\section{Valoración de tecnología en Colombia: casos seleccionados}

Sin pretender desconocer los posibles desarrollos en el tema de valoración tecnológica en Colombia, dado que para este artículo no se efectuó una revisión exhaustiva sobre el asunto, a continuación se referencian dos líneas en las que trabaja el grupo de investigación al cual pertenecen los autores de este documento, que pueden considerarse como enfoques alternativos a los tradicionales. La primera línea se refiere a la valoración de capacidades tecnológicas y la segunda a la valoración tecnológica desde la perspectiva biológica.

\section{Valoración de capacidades tecnológicas}

Considerando la definición de tecnología desde su perspectiva más amplia, que de acuerdo con Angelo et al. (2008) no se limita a tecnologías individuales, sino que abarca la capacidad tecnológica total de la empresa, el trabajo de Cristancho (20II) propone la valoración de una tecnología en el Instituto Nacional de Cancerología de Colombia (INC) a través 
de la medición de las capacidades tecnológicas logradas por su implementación.

Este trabajo valoró la radiofarmacia en el INC, entendiendo el radiofármaco como una sustancia química que posee átomos radioactivos dentro de su estructura y es apropiado para la administración a humanos, formulado en varias for- mas químicas y físicas para depositar su radioactividad en partes específicas del organismo con fines diagnóstico o en el tratamiento de enfermedades como el cáncer. La mecánica para el diagnóstico se logra con la radiación gamma que emiten estas moléculas, la cual penetra los tejidos y escapa del cuerpo, permitiendo así la detección externa y su medida (Henderson, 2002). La radiofarmacia hospitalaria del INC

\begin{tabular}{|c|c|c|c|c|c|}
\hline \multirow{3}{*}{$\begin{array}{c}\text { Niveles de } \\
\text { Capacidades }\end{array}$} & \multicolumn{4}{|c|}{ Funciones técnicas primarias } & \multirow{3}{*}{$\begin{array}{c}\begin{array}{c}\text { Función técnica de } \\
\text { soporte }\end{array} \\
\text { Vínculos e interacción } \\
\begin{array}{c}\text { Vinculación } \\
\text { externa }\end{array} \\
\end{array}$} \\
\hline & \multicolumn{2}{|c|}{ Inversión } & \multicolumn{2}{|c|}{ Producción } & \\
\hline & $\begin{array}{l}\text { Toma de } \\
\text { decisiones y } \\
\text { control }\end{array}$ & $\begin{array}{l}\text { Preparación y } \\
\text { ejecución de } \\
\text { proyectos }\end{array}$ & $\begin{array}{c}\text { Centrada en los } \\
\text { procesos y en la org. } \\
\text { de la producción }\end{array}$ & $\begin{array}{l}\text { Centrada en } \\
\text { el producto }\end{array}$ & \\
\hline \multicolumn{6}{|c|}{ Capacidades rutinarias de producción: capacidades para usar y operar la tecnología existente } \\
\hline $\begin{array}{l}\text { Capacidades } \\
\text { operativas } \\
\text { básicas }\end{array}$ & Logrado & Logrado & Logrado & Logrado & $\begin{array}{l}\text { En desarrollo para la } \\
\text { producción de nucleidos } \\
\text { (formas inestables de un } \\
\text { elemento que liberan } \\
\text { radiación a medida que se } \\
\text { descomponen y se vuelven } \\
\text { más estables) con el } \\
\text { ciclotrón (acelerador de } \\
\text { partículas). }\end{array}$ \\
\hline $\begin{array}{l}\text { Apropiación de } \\
\text { capacidades }\end{array}$ & Organizacional & Organizacional & $\begin{array}{l}\text { Organizacional } \\
\text { Personal }\end{array}$ & Organizacional & Organizacional \\
\hline \multicolumn{6}{|c|}{ Capacidades tecnológicas innovativas: capacidades para generar y administrar el cambio técnico } \\
\hline $\begin{array}{c}\text { Capacidades } \\
\text { innovativas } \\
\text { básicas } \\
\end{array}$ & Logrado & Logrado & Logrado & Logrado & Logrado \\
\hline & $\begin{array}{l}\text { Organizacional } \\
\text { débil }\end{array}$ & & & $\begin{array}{l}\text { Organizacional } \\
\text { débil }\end{array}$ & \\
\hline $\begin{array}{l}\text { Apropiación de } \\
\text { capacidades }\end{array}$ & $\begin{array}{l}\text { En proceso de } \\
\text { consolidación en } \\
\text { el nivel } \\
\text { organizacional }\end{array}$ & Organizacional & Organizacional & $\begin{array}{l}\text { Fuerte en } \\
\text { personal, en } \\
\text { actores } \\
\text { relacionados con } \\
\text { entes reguladores }\end{array}$ & Organizacional \\
\hline $\begin{array}{l}\text { Capacidades } \\
\text { innovativas } \\
\text { intermedias }\end{array}$ & Logrado & Logrado & Logrado & Logrado & Logrado \\
\hline $\begin{array}{l}\text { Apropiación de } \\
\text { capacidades }\end{array}$ & $\begin{array}{l}\text { Organizacional } \\
\text { débil en factores } \\
\text { de vigilancia } \\
\text { tecnológica } \\
\text { Fuertemente } \\
\text { personal en } \\
\text { actores externos a } \\
\text { la organización }\end{array}$ & Organizacional & Organizacional débil & $\begin{array}{l}\text { Organizacional } \\
\text { débil } \\
\text { Fuerte en } \\
\text { personal, en } \\
\text { actores } \\
\text { relacionados con } \\
\text { entes reguladores }\end{array}$ & $\begin{array}{l}\text { Organizacional débil en los } \\
\text { factores en que se } \\
\text { desarrolla. } \\
\text { Ausencia de criterios } \\
\text { organizacionales para la } \\
\text { transferencia de tecnología. }\end{array}$ \\
\hline $\begin{array}{c}\text { Capacidades } \\
\text { innovativas } \\
\text { avanzadas }\end{array}$ & No se desarrollará & $\begin{array}{l}\text { Logrado para la } \\
\text { producción de } \\
\text { nucleídos por } \\
\text { ciclotrón }\end{array}$ & $\begin{array}{l}\text { Se desarrollará para } \\
\text { la producción de } \\
\text { nucleídos por } \\
\text { ciclotrón }\end{array}$ & $\begin{array}{l}\text { Se desarrollará } \\
\text { para la } \\
\text { producción de } \\
\text { nucleídos por } \\
\text { ciclotrón } \\
\end{array}$ & $\begin{array}{l}\text { Se desarrollará para la } \\
\text { producción de nucleídos por } \\
\text { ciclotrón }\end{array}$ \\
\hline $\begin{array}{l}\text { Apropiación de } \\
\text { capacidades }\end{array}$ & No aplica & Organizacional & & & \\
\hline
\end{tabular}

Tabla I. Capacidades tecnológicas alcanzadas mediante la radiofarmacia del INC. Fuente: adaptado de Dutrénit et al. (2002) y Bell y Pavitt (1995) por Cristancho (20II).

ISSN: 07 I8-2724. (http://www.jotmi.org)

Journal of Technology Management \& Innovation (c) Universidad Alberto Hurtado, Facultad de Economía y Negocios. 
está proyectada para generar y fortalecer la investigación en oncología y medicina nuclear produciendo radiofármacos que incrementen la eficacia del diagnóstico, tratamiento y paliación del cáncer (INC, 2007). Su producción en la mayoría de los casos debe ser realizada rápidamente, antes de la administración al paciente debido al corto tiempo de estabilidad del radio núcleo, lo cual limita su producción a nivel industrial, además tiene como objetivo transversal centralizar el manejo de fuentes abiertas de radiación, asegurando la adecuada manipulación de material radioactivo en todas las etapas del proceso.

Con base en el análisis sobre la complejidad administrativa, tecnológica y de relacionamiento con la cual se implementó la radiofarmacia en el INC, se seleccionó la matriz desarrollada por Bell y Pavitt (1995), por ser aplicable a las dinámicas tecnológicas observadas en la organización, y además porque esta herramienta, con bajos niveles de profundidad, brinda facilidad de aplicación.

De acuerdo con el resumen del estudio presentado en la Tabla I, en el nivel de capacidad operativa básica, la radiofarmacia generó en el INC la capacidad de asimilar la tecnología y replicar las metodologías de producción básicas. En cuanto a las capacidades de innovación básicas, la radiofarmacia permitió a la organización lograr la capacidad de hacer cambios incrementales de proceso para mejorar la calidad. Respecto a las capacidades intermedias de innovación, la implementación de esta tecnología generó habilidades plenas de producción y la capacidad de innovación de procesos y diseño de productos. En el nivel de las capacidades avanzadas de innovación, la tecnología analizada facilitó que el INC realice actividades propias de I+D de productos y procesos vinculados a las necesidades del mercado. Se puede evidenciar que la organización consolidó la mayoría de capacidades tecnológicas con la implementación de la radiofarmacia, con base principalmente en la complejidad técnica requerida para el desarrollo de esta tecnología. Es de resaltar que la variedad de técnicas necesarias para la implementación de la tecnología obligó al INC a diversificar sus fuentes de asesoría y aprovisionamiento de la tecnología, para lo cual se gestionó el apoyo del Organismo Internacional de Energía Atómica - OIEA, de los proveedores internacionales de esta tecnología, y de los organismos nacionales encargados de promover el uso de la energía atómica con fines pacíficos. En este caso, la tecnología se valoró por su impacto en la organización al contribuir directamente a la generación de capacidades que fortalecen su desempeño y otorgan mayor competitividad a través del uso y aprovechamiento de los recursos tecnológicos.

\section{Consideraciones para un abordaje de la valoración tecnológica desde la biología}

Los métodos y modelos de valoración tecnológica han sido cuestionados en diferentes épocas, por considerarse incompletos y desconocer aspectos relevantes como el conocimiento incorporado en los activos tecnológicos, su complejidad y el entorno en el que dichos activos se encuentran. Hunt et al. (2003) afirman que la valoración de tecnología sigue siendo un arte más que una ciencia, ya que los métodos utilizados han sido adaptados de los que se aplican para valorar otro tipo de activos más tradicionales, gracias a la flexibilidad gerencial, por lo cual aún existe un campo importante para la investigación en esta área. Eichner Gemünden y Kautzsch (2007) plantean que si bien son bastante conocidos los métodos clásicos de valoración de inversiones, tales como el flujo de caja descontado, estos tienden a fallar en el ámbito tecnológico, dado que las inversiones en tecnología no solo tienen un futuro incierto, sino que su plan de negocio es flexible y sujeto a cambios, por lo que a menudo la decisión de inversión se basa en una mera cuestión de criterio.

Kaplan (1986), Kakati y Dhar (1991) y Raafat (2002) han sugerido involucrar factores estratégicos, como los cualitativos, de manera más explícita en los procesos de valoración tecnológica, e incluso se ha recomendado el uso de aproximaciones mixtas o híbridas en las que se consideren tanto factores cuantitativos como cualitativos. Small y Chen (1997, citado en Doerr, Gates y Mutty, 2006) empíricamente evidenciaron que muchas empresas no usan técnicas de evaluación sofisticada, pero aquellas que emplean una aproximación amplia incluyendo aspectos cuantitativos y cualitativos, tienden a lograr mejores resultados en la implementación de la tecnología valorada. Adicionalmente, como uno de los retos de la gestión tecnológica en el marco de la sostenibilidad industrial, Paramanathan et al. (2004) han identificado la necesidad de nuevos métodos de valoración tecnológica que integren el concepto de triple cuenta de resultados, en sus dimensiones económica, medioambiental y social.

Tomando en consideración estas críticas, los autores de este artículo están desarrollando una propuesta sobre una aproximación que busca aportar a la actualización del tema de valoración tecnológica teniendo como base la visión organicista, de manera que se agreguen al proceso atributos como la dinamicidad, la complejidad y el carácter sistémico a partir de elementos y conceptos biológicos, consolidando un referente conceptual que responda a la necesidad de dar mayor alcance y amplitud a los procesos de valoración tecnológica en las organizaciones, así como pertinencia a contextos como el colombiano, lo cual no ha sido evidenciado en la literatura sobre el tema que se revisó para este documento. 
El problema de la visión mecanicista y su crisis ha sido afrontado en gran parte mediante el paradigma organicista en diferentes ámbitos. Es el caso del avance experimentado en la concepción de la organización, relacionado con lo que Capra (1998) califica como un cambio de paradigma social, en el cual se pasó de un marco de ideas que prevaleció por centenares de años, a un nuevo paradigma en donde se adopta una visión holística y ecológica del mundo, por lo cual la organización se comenzó a entender como un sistema abierto, al igual que los organismos biológicos. Según Morgan (1998), se recurrió a la biología como una fuente nueva de ideas para estudiar e identificar las necesidades organizacionales, ante la crisis de la visión mecanicista.

Este enfoque organicista también ha impactado a la ingeniería y la gestión tecnológica, a través de imitaciones del comportamiento de los organismos vivos y otros elementos biológicos tomados como soporte para el planteamiento de conceptos y el desarrollo de herramientas y técnicas que han permitido una mejor operacionalización, particularmente de la gestión de manufactura (Jiménez y Castellanos, 2008; Jiménez, Castellanos y Montoya, 2009). Como ejemplo está la aplicación de agentes inteligentes autónomos en sistemas heterárquicos (en red) de manufactura (Maione y Naso, 2002; 2003; 2004), el desarrollo de sistemas de manufactura flexible y sistemas inteligentes con características como la autoorganización, la adaptación eficiente y la evolución (Brezocnik y Balic, 200I; Brezocnik, Balic y Brezocnik, 2003) y la conceptualización e implementación de sistemas biológicos de manufactura, cuyo modelamiento se inspira en el comportamiento, atributos y estructuras de los organismos vivos (Ueda, Vaario y Ohkura, 1997; Ueda et al., 2000; 200I).

La valoración tecnológica, como una de las funciones fundamentales de la gestión tecnológica y tema clave en los procesos industriales y organizacionales, puede complementar su enfoque tradicional con nuevos abordajes, como el que plantea la visión organicista, a partir de elementos y conceptos biológicos. Entendiendo la valoración tecnológica como un proceso, la filosofía de procesos puede ser tomada como base para un análisis conceptual; esta rama de la filosofía señala que la existencia natural es mejor entendida en términos de procesos en lugar de cosas, o modos de cambio en lugar de estabilidades fijas; el cambio - ya sea físico, orgánico, sicológico, etc. - es un rasgo predominante de lo real (Rescher, 2008). Al analizar la valoración tecnológica en términos de cambio, se busca abarcar características como la flexibilidad y la adaptación, puesto que esta requiere responder a las dinámicas del proceso de adquisición (o generación), asimilación y dominio de los activos tecnológicos por parte de la organización, y esto ocurre en función del tiempo.
Así mismo, se propone recurrir a elementos biológicos para complementar la valoración tecnológica y aportar en su dinamización, tomando en cuenta que en el caso de los organismos vivos, así como en la tecnología, no siempre es aplicable el enfoque mecanicista, con el determinismo y el reduccionismo como base, para el estudio de este tipo de sistemas, caracterizados por su complejidad. Pero el estudio de los organismos biológicos también se ha visto influenciado tanto por el reduccionismo entendido como forma de pensamiento, como por el reduccionismo epistemológico u ontológico, que afirma que el lenguaje de una disciplina puede ser traducido al lenguaje de otra disciplina básica (Katz, 20I0). Se argumenta que la reducción permite establecer un nexo racional entre las proposiciones de una teoría determinada con distinto grado de generalidad, por lo que este proceso se transforma en una necesidad para el desarrollo de la propia teoría en un campo concreto del conocimiento humano (Díaz y Calzadilla, 200I).

En el caso de la biología, de acuerdo con Caponi (2004) se hace necesaria una distinción entre la ramas funcional y evolutiva de la biología, cuando se trata de su reducción al lenguaje de la física y la química, como una de las cuestiones principales en la filosofía de la biología. Este autor afirma que la biología funcional puede reducirse al lenguaje de la física, ya que las respuestas frente a sus objetos cognitivos pueden escribirse en dicho lenguaje. Empero, las cuestiones de la biología evolutiva son intraducibles a la física o la química. Por tanto, como vía para aportar a la valoración tecnológica, en la investigación en curso se considera que la biología evolutiva tiene mayor pertinencia frente al pensamiento reduccionista y determinista prevaleciente en el estudio tanto de la tecnología como de los organismos biológicos. Igualmente la filosofía de procesos permite ratificar la biología evolutiva como el referente que más se ajusta para el análisis y complementación de la valoración tecnológica.

\section{Conclusiones}

La valoración tecnológica es una temática en desarrollo, en donde el propio concepto está evolucionando, llegando a entenderse por valoración la determinación no solo del valor de un bien, sino también de su impacto en la organización que lo adquiere o genera. En términos de tecnología, los procesos de valoración deben considerar tanto las técnicas tradicionales orientadas a bienes tangibles representados por el componente duro del paquete tecnológico, como a nuevos enfoques para valorar su componente blando y en general, los activos intangibles. Así mismo, se encuentra en la literatura una gran cantidad de propuestas en torno a métodos y técnicas de valoración de tecnologías, cuya elección está supeditada al objetivo que se persiga y debe tomar en cuenta la pertinencia de la técnica de valoración para el contexto en el que pretende aplicarse, así como la viabilidad de 
realización del análisis, principalmente por la disponibilidad de información. También es posible el uso de varios métodos simultáneamente.

La investigación sobre valoración tecnológica se ha enfocado solo en algunas de las dimensiones y características de la tecnología, por lo cual ha sido criticada y analizada por diversos autores, en búsqueda de mayor amplitud y alcance. En este artículo se identificaron varias investigaciones que han buscado plantear nuevas alternativas al abordaje tradicional de la valoración tecnológica, que ha sido principalmente económico o monetario, y se evidencia que la mayoría de estos trabajos se orienta a la valoración del impacto social, de manera que se otorgue a los tomadores de decisiones mejores elementos al momento de seleccionar y adquirir una tecnología. Empero, estos enfoques no integran otras dimensiones de la tecnología, al basarse generalmente en aspectos cualitativos y subjetivos. También se encontraron otros trabajos que buscan nuevos métodos para valorar la tecnología desde la perspectiva económica, partiendo de las deficiencias de los métodos tradicionales de valoración empleados habitualmente en la evaluación financiera de proyectos. Estos desarrollos pretenden considerar el riesgo y la incertidumbre especialmente de tecnologías emergentes. En este caso, la dimensión es fundamentalmente monetaria, y no abarca otros aspectos de la tecnología.

En el caso colombiano, si bien no se profundizó en el análisis de los desarrollos y aplicaciones de la valoración tecnológi$\mathrm{ca}$, se destaca que es posible valorar una tecnología específica en un contexto particular a partir de su impacto en la organización a través de la generación de capacidades para aprovechar dicha tecnología. Este hecho resulta relevante en el contexto de los países en desarrollo, tomando en cuenta que la asimilación tecnológica y la creación de capacidades en tal proceso son claves en la reducción de las brechas frente a los países desarrollados.

Por otra parte, a partir de lo hallado en la literatura, puede concluirse que el enfoque prevaleciente de valoración tecnológica se caracteriza por ser fundamentalmente transaccional, estático y altamente heterogéneo en métodos, técnicas y modelos. Con base en este hallazgo, en este trabajo se plantea una propuesta que busca enriquecerlo al retomar elementos biológicos como la complejidad, la dinamicidad, la flexibilidad y la adaptabilidad, y asociarlos a la tecnología para determinar su valor de manera más integral, considerando tanto los aspectos tangibles como los intangibles de los bienes tecnológicos.

Se busca que dicho aporte pueda ser aplicable de manera sencilla pero confiable en ámbitos como los sectores tradicionales y las pequeñas y medianas empresas que caracterizan la economía colombiana, en donde generalmente no se cuenta con información completa ni suficientemente codificada, además de que no siempre es de tipo cuantitativo. La validez de este enfoque parte de la destacada contribución de la biología a la gestión organizacional y tecnológica.

\section{Referencias}

AïT-EL-HADJ, S. (1990). Gestión de la tecnología. La empresa frente a la mutación tecnológica. Ediciones Gestión 3000, Estados Unidos.

ANGELO, R., Domenico, C., Luigi, I. y lacobelli, M. (2008). Technology Valuation for Biotechnology: techniques for valuation of Intellectual Property. IBIC Conference, Italia.

BELL, M. (2007). Types and Levels of Technological Capability in Developing Countries. Background Paper No. I0. In Technological learning and the development of production and innovative capacities in the industry and infrastructure sectors of the least developed countries: what roles for ODA? (PP. I00-I I I).SPRU-Science and Technology Policy Research, University of Sussex, Paper prepared for UNCTAD Division for Africa, Least Developed Countries and Special Programmes, Brighton, United Kingdom.

BELL, M. y Pavitt, K. (1995). The development of technological capabilities. In Trade, technology and international competitiveness (pp. 69-10I).The World Bank, Washington.

BLACK, F. y Scholes, M. (1973). The pricing of options and corporate liabilities. The journal of political economy,(8I), 637-654.

BONTIS, N. (200I). Assessing knowledge assets: a review of the models used to measure intellectual capital. International Journal of Management Reviews, 3(I), 4I-60.

BRENT, A. C. y Kruger, W. J. L. (2009). Systems analyses and the sustainable transfer of renewable energy technologies: A focus on remote areas of Africa. Renewable Energy, 34(7), |774-|78I.

BRENT, A. C. y Rogers, D. E. (2010). Renewable rural electrification: Sustainability assessment of mini-hybrid off-grid technological systems in the African context. Renewable Energy, 35(I), 257-265.

BREZOCNIK, M. y Balic, J. (200I).A genetic-based approach to simulation of self-organizing assembly. Robotics and Computer-Integrated Manufacturing, I7(I-2), II3-I 20.

BREZOCNIK, M., Balic, J.y Brezocnik, Z. (2003). Emergence of intelligence in next-generation manufacturing systems. Robotics and Computer-Integrated Manufacturing, I9(I-2), 55-63.

ISSN: 07 I8-2724. (http://www.jotmi.org) 
CAPONI, G. (2004). El reduccionismo en la biología contemporánea. Signos filosóficos, I2(2), 33-62.

CAPRA, F. (1998). La Trama de la Vida, Una Perspectiva Nueva de los Sistemas Vivos. Editorial Anagrama, Barcelona.

CHIESA,V., Gilardoni, E. y Manzini, R. (2005). The valuation of technology in buy-cooperate-sell decisions. European Journal of Innovation Management, 8(I), 5-30.

COWAN, K. R.y Daim,T.U. (2009). Comparative technological road-mapping for renewable energy. Technology in Society, 3 I (4), 333-34I.

CRISTANCHO, A. (20II). Valoración de las Capacidades y Competencias Tecnológicas. Consideraciones para su Aplicación en el Aparato Productivo Colombiano. Trabajo para optar al título de Magíster en ingeniería industrial, Universidad Nacional de Colombia.

DAHLMAN, C. y Westphal, L. (1982). Technological effort in industrial development: an interpretative survey of recent research. World Bank

DAIM,T. U. e Intarode, N. (2009).A framework for technology assessment: Case of a Thai building material manufacturer. Energy for Sustainable Development, 13(4), 280-286.

DAIM, T. U. y Cowan, K. R. (20I0). Assessing renewable energy portfolio futures with multiple perspectives: The case of the northwest US. Technology in Society, 32(4), 255-263.

DAIM,T. U., Yates, D., Peng,Y.y Jimenez, B. (2009). Technology assessment for clean energy technologies: The case of the Pacific Northwest. Technology in Society, 3 I (3), 232-243.

DÍAZ,V. P. y Calzadilla, A. (200I). El Reduccionismo, Antirreduccionismo y el Papel de los Enfoques y Métodos Generales del Conocimiento Científico. Cinta de Moebio, II, 148-160.

DOERR, K. H., Gates, W. R. y Mutty, J. E. (2006). A hybrid approach to the valuation of RFID/MEMS technology applied to ordnance inventory. International Journal of Production Economics, I03(2), 726-74I.

DUBIN, J. A. (2007). Valuing intangible assets with a nested logit market share model. Journal of econometrics, I39(2), 285-302.

DUTKIEWICZ, V. A., Qureshi, S., Husain, L., Schwab, J. J. y Demerjian, K. L. (2006a). Elemental composition of PM2.5 aerosols in Queens, New York: Evaluation of sources of fineparticle mass. Atmospheric Environment, 40(Supplement 2), 347-359.
DUTKIEWICZ, V. A., Qureshi, S., Khan, A. R., Husain, L., Schwab, J. J. y Demerjian, K. L. (2006b). Field test data for 42 liter per minute PM2.5 aerosol sampler used during the PMTACS-NY intensives held at Queens College, Queens, NY.Atmospheric Environment, 40(Supplement 2), I82-191.

DUTRÉNIT, G.,Vera-Cruz,A.,Arias,A.,Avendaño, G., Gil, J. L., Sampedro, J. L. et al. (2002). Marco analítico para el análisis de los procesos de acumulación de capacidades tecnológicas. Colef/Flacso/UAM, México.

EDVINSSON, L. y Sullivan, P. (1996). Developing a model for managing intellectual capital. European Management Journal, 14(4).

EICHNER, T., Gemünden, H. G. y Kautzsch, T. (2007). What is Technology Worth? The Journal of Investing, 16(3), 96-103.

ELÓl, D. y Santiago, L. (2008). Avaliar X valorar novas tecnologias: desmistificando conceitos.www.institutoinovacao. com.br/downloads/Avaliar_x_Valorar_I.pdf. [Accessed 2-520II]

FARRUKH, C., Dissel, M., Jackson, K., Phaal, R. y Probert, D. (2009). Valuing technology along a timeline of technological maturity. International Journal of Technology Management, 48(I), 42-55.

FLEISCHER, T., Decker, M. y Fiedeler, U. (2005). Assessing emerging technologies--Methodological challenges and the case of nanotechnologies. Technological Forecasting and Social Change, 72(9), I I I 2-I I 2 I.

HENDERSON, D. (2002). Radiofarmacia - Parte I. Programa de Entrenamiento Asistido a Distancia para Tecnólogos en Medicina Nuclear. Hospital Westmead, Sydney- OIEA AusAID, Australia.

HETMAN, F. (1973). Society and the Assessment of Technology. Organization for Economic Cooperation and Development - OECD, París.

HILTY, L. M., Arnfalk, P., Erdmann, L., Goodman, J., Lehmann, M. y Wâ€žger, P.A. (2006). The relevance of information and communication technologies for environmental sustainability - A prospective simulation study. Environmental Modelling \& Software, 2I(II), I6I8-1629.

HUNT, F. H., Probert, D. R., Wong, J. C. y Phaal, R. (2003). Valuation of technology: exploring a practical hybrid model. PICMET (pP. 47-53), IEEE.

ISSN: 07 I8-2724. (http://www.jotmi.org)

Journal of Technology Management \& Innovation (c) Universidad Alberto Hurtado, Facultad de Economía y Negocios. 
HUNT, F. H., Thorn, V., Mitchell, R., Probert, D. y Phaal, R. (2007). Internal technology valuation: Real world issues. PICMET (pp. I736- 1742), IEEE.

INC. (2007). Nuevas perspectivas de la radiofarmacia hospitalaria: Radiofarmacia y Central de Mezclas para el Instituto Nacional de Cancerología E.S.E. INC, Bogotá.

JANSSEN, M. C. W. y Mendys-Kamphorst, E. (2007). Evolution of market shares with repeated purchases and heterogeneous network externalities. Journal of Evolutionary Economics, I7(5), 55I-577.

JIMÉNEZ, C. N. y Castellanos, O. (2008). Perspectivas y con sideraciones sobre el aporte biológico al desarrollo de la gestión de tecnología. Innovar, 18(32), 47-64.

JIMÉNEZ, C. N. y Castellanos, O. (20I I). Enfoque multidimensional y dinámico de la valoración tecnológica. Sexto congreso internacional de la Red de Investigación y Docencia sobre Innovación Tecnológica RIDIT, Manizales.

JIMÉNEZ, C. N. y Castellanos, O. (20I2). Technology and its value at the Bottom of the Pyramid. Portland International Conference on Management of Engineering and Technology (pp. 1355-1360), PICMET, Vancouver, Canadá.

JIMÉNEZ, C. N., Castellanos, O. y Montoya, A. (2009). Manufactura biológica e inteligente: atributos de la vida aplicados al desarrollo tecnológico. Ingeniería e investigación, 29(2), |27-|34.

KAKATI, M. y Dhar, U. R. (I99I). Investment justification in flexible manufacturing systems. Engineering Costs and Production Economics, 2 I (3), 203-209.

KAPLAN, R. S. (1986). Must CIM be justified by faith alone? Harvard Business Review,(March-April), 87-95.

KATZ, M. (2010). El reduccionismo. Epistemología e Historia de la Química - Curso 2010. Argentina, Universidad Nacional de Tucumán.

KOIVISTO, R., Wessberg, N., Eerola, A., Ahlqvist, T., Kivisaari, S., Myllyoja, J. et al. (2009). Integrating future-oriented technology analysis and risk assessment methodologies. Technological Forecasting and Social Change, 76(9), I I63-I I 76.

LAI,Y. H. y Che, H. C. (2009). Modeling patent legal value by Extension Neural Network. Expert Systems with Applications, 36(7), 10520-10528.

LALL, S. (1992). Technological capabilities and industrialization.World Development, 20(2), I65-I86.
LES. (2008). Technology valuation and License negotiation course. The Licensing Executives Society International.

LEWIS, N., Enke, D. y Spurlock, D. (2004). Valuation for the Strategic Management of Research and Development Projects:The Deferral Option. Engineering Management Journal, 16(4), 36-48.

LI,Y. R. y Chen, Y. G. (2006). Managing Technology: The Technology Valuation Approach. PICMET (pp. 535-540), IEEE, Turquía.

MAIONE, G. y Naso, D. (2002). Adaptation of multi-agent manufacturing control by means of genetic algorithms and discrete event simulation.:Vol. 4. Proceedings of the IEEE International Conference on Systems, Man and Cybernetics (pp. 529-534).

MAIONE, G. y Naso, D. (2003).A genetic approach for adaptive multiagent control in heterarchical manufacturing systems. IEEE Transactions on Systems, Man, and Cybernetics Part A:Systems and Humans., 33(5), 573-588.

MAIONE, G. y Naso, D. (2004). Modelling adaptive multiagent manufacturing control with discrete event system formalism. International Journal of Systems Science, 35(10), $59|-6| 4$.

MALONEY, J. D. (1982). How companies assess technology. Technological Forecasting and Social Change, 22(3-4), 32I329.

MORGAN, G. (1998). Imágenes de la organización. Alfaomega, México.

MUSANGO, J. K. y Brent, A. C. (20 I Ia). A conceptual framework for energy technology sustainability assessment. Energy for Sustainable Development, I5(I), 84-9I.

MUSANGO, J. K. y Brent, A. C. (20I Ib). Assessing the sustainability of energy technological systems in Southern Africa:A review and way forward. Technology in Society, 33(I), I45-I55.

NYOKA, M. y Brent, A. C. (2007). Application of an environmental valuation approach that incorporates externality costs in sustainability decision-making of the metallurgical sector. Journal of The South African Institute of Mining and Metallurgy, I07(10), 663-669.

PARAMANATHAN, S., Farrukh, C., Phaal, R. y Probert, D. (2004). Implementing industrial sustainability: the research issues in technology management. R \& D Management, 34(5), 527-537. 
PARK, Y. y Park, G. (2004). A new method for technology valuation in monetary value: procedure and application. Technovation, 24(5), 387-394.

PRAHALAD, C. K. y Hamel, G. (1990). The core competence of the corporation. Harvard Business Review,(May-June), 7991.

QURESHI, S., Dutkiewicz,V.A., Khan, A. R., Swami, K., Yang, K. X., Husain, L. et al. (2006). Elemental composition of PM2.5 aerosols in Queens, New York: Solubility and temporal trends. Atmospheric Environment, 40(Supplement 2), 238251 .

RAAFAT, F. (2002). A comprehensive bibliography on justification of advanced manufacturing systems. International Journal of Production Economics, 79(3), 197-208.

REN, X., Harder, H., Martinez, M., Lesher, R. L., Oliger,A., Simpas, J. B. et al. (2003). OH and HO2 Chemistry in the urban atmosphere of New York City. Atmospheric Environment, 37(26), 3639-365I.

RESCHER, N. (2008). Process Philosophy. In E. E.N.Zalta (Ed.), The Stanford Encyclopedia of Philosophy.The Metaphysics Research Lab, Stanford University, Stanford, CA.

ROBINSON, D. K. R. y Propp, T. (2008). Multi-path mapping for alignment strategies in emerging science and technologies. Technological Forecasting and Social Change, 75(4), 5I7-538.

SÁBATO, J. (1979). Ensayos en Campera. Juárez Editores, Buenos Aires.

SHEHABUDDEEN, N., Probert, D. y Phaal, R. (2006). From theory to practice: challenges in operationalising a technology selection framework. Technovation, 26(3), 324-335.

TAVANA, M. y Sodenkamp, M.A. (2010). A fuzzy multi-criteria decision analysis model for advanced technology assessment at Kennedy Space Center. Journal of the Operational Research Society, 61 (I0), I459-I470.

TAYLOR, F.W. (I9| I). Scientific management. New York.

THORN, V., Hunt, F. H., Mitchell, R., Probert, D. y Phaal, R. (20II). Internal technology valuation: Real world issues. International Journal of Technology Management, 53(2-4), I49160.

TIDD, J. (2000). From knowledge Management to Strategic Competence. University of Sussex, UK.
TRAN, T. A. y Daim, T. (2008). A taxonomic review of methods and tools applied in technology assessment. Technological Forecasting and Social Change, 75(9), I396- 1405.

UEDA, K., Hatono, I., Fujii, N. y Vaario, J. (2000). Reinforcement learning approaches to Biological Manufacturing System. CIRP Annals - Manufacturing Technology. 49, 343-346. 1920.

UEDA, K., Hatono, I., Fujii, N. y Vaario, J. (200I). Line-less production system using self-organization:A case study for BMS. CIRP Annals - Manufacturing Technology. 50, 319-322. 1922.

UEDA, K., Vaario, J. y Ohkura, K. (1997). Modelling of biological manufacturing systems for dynamic reconfiguration. CIRP Annals - Manufacturing Technology. 46, 343-X72. 1922.

VALHONDO, D. (2003). Gestión del conocimiento: del mito a la realidad. Ediciones Díaz de Santos

WAISSBLUTH, M. (1990). El paquete tecnológico y la innovación. In Conceptos Generales de Gestión Tecnológica. Colección Ciencia y Tecnología No. 26.

WATKINS, W. M. (1998). Technology and business strategy: getting the most out of technological assets. Greenwood Press, Wesport, USA.

WEIMER, S., Drewnick, F., Hogrefe, O., Schwab, J. J., Rhoads, K., Orsini, D. et al. (2006). Size-selective nonrefractory ambient aerosol measurements during the Particulate Matter Technology Assessment and Characterization Study. Journal of Geophysical Research-Atmospheres, I I I (DI8). 